Disclosure of Interest: None declared

DOI: 10.1136/annrheumdis-2017-eular.2513

\section{FRIDAY, 16 JUNE 2017}

\section{Vasculitis}

\section{FRI0303 THE IGG4:IGG RNA RATIO IS A NEW AND PROMISING DISEASE ACTIVITY MARKER IN GRANULOMATOSIS WITH POLYANGIITIS}

A. Al-Soudi ${ }^{1}$, M. Doorenspleet ${ }^{1}$, R. Esveldt ${ }^{1}$, S. Tas ${ }^{1}$, R. van Vollenhoven ${ }^{1}$, P. Klarenbeek ${ }^{1}$, N. de Vries ${ }^{1,2}$. ${ }^{1}$ Amsterdam Rheumatology and Immunology Center ${ }^{2}$ Department of Experimental Immunology, Academic Medical Center Amsterdam, Netherlands

Background: Granulomatosis with Polyangiitis (GPA) is a form of vasculitis characterized by inflammation of blood vessels in lungs, kidneys and the ear, nose and throat region. Regular monitoring and treatment adjustments are common, as the disease activity tends to fluctuate over time. Unfortunately, good markers for disease activity are lacking. This leads to both over- and undertreatment. Immunoglobulin G4 positive (IgG4+) B-cells and plasma cells are implicated in the pathogenesis of GPA, but the level of serum IgG4 does not seem to be a good disease activity marker. Recently we developed a test that indirectly measures the presence of IgG4+ B-cells/plasma cells by measuring the IgG4:IgG RNA ratio ${ }^{1}$. We hypothesized that this test could be used as disease activity marker.

Objectives: To test the IgG4:IgG RNA ratio in peripheral blood as a disease activity marker in GPA.

Methods: 27 PR3+ ANCA+ positive GPA patients were included in this crosssectional study. Mean age was 52 years, $56 \%$ were female, and $39 \%$ had active disease. For each patient the ESR, CRP, BVAS, and ANCA titre were measured and peripheral blood samples were obtained. Patients were defined as having active disease if the BVAS was $\geq 3$. In addition we included 10 healthy controls, and 63 patients with other immune mediated inflammatory diseases (systemic lupus erythematosus (SLE) $(n=24)$, rheumatoid arthritis (RA) $(n=19)$, primary sclerosing cholangitis (PSC) $(n=20)$ ). A validated qPCR test was performed in all groups to measure the IgG4:IgG RNA ratio in peripheral blood samples ${ }^{1}$

Results: The median IgG4:IgG RNA ratio was significantly higher in the GPA cohort $(5.7$, IQR 2.6 - 19.7) compared to all control groups: 1.2 in SLE (0.7 3.3; $p<0.01), 2.5$ in RA $(0.7-4.1 ; p<0.05), 1.6$ in PSC $(1.0-2.8 ; p<0.001)$ and 1.3 in $\mathrm{HC}(0.6-1.8, \mathrm{p}<0.01)$. In addition, the median $\lg 44: \lg$ RNA ratio was significantly higher in patients with active disease (23.8; IQR $12.1-29.1)$ compared to patients in remission $(3.5$; IQR $2.0-5.5)(p<0.0001)$. The height of the lgG4:IgG RNA ratio significantly correlated with height of the BVAS $\left(r^{2}=0.76\right.$, $\mathrm{p}<0.0001$ ), while the ESR, CRP and ANCA titre did not. Interestingly, IgG4/lgG RNA ratios among patients with active disease were consistently above 9.3, and among patients in remission they were below this threshold.

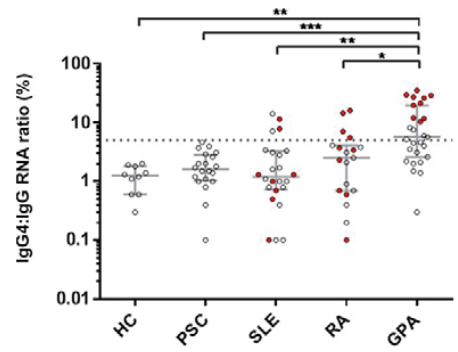

IgG4:IgG RNA ratio (\%) in healthy controls (HC), primary sclerosing cholangitis (PSC), systemic lupus erythematosus (SLE), rheumatoid arthritis (RA) and granulomatosis with polyangiitis (GPA). Red dots represent patients with active disease (resp. SLEDAI, DAS28 and BVAS). ${ }^{*}<0.05,{ }^{* *}<0.01,{ }^{\star \star *}<0.001$

Conclusions: The IgG4:IgG RNA ratio distinguishes active GPA from GPA in remission with excellent specificity and sensitivity. Moreover the ratio shows a significant correlation with disease severity, in contrast to ESR, CRP and ANCA titre. Retesting in another, prospective study is indicated to validate the $\lg$ G4:lgG ratio as a novel, highly sensitive and specific marker of disease activity in GPA. References:

[1] Doorenspleet ME et al. Hepatology 2016; 64(2): 501-7.

Acknowledgements: We thank D. van der Coelen for the technical assistance, the doctors of the vasculitis outpatient clinic, especially Dr. B.J.H. van der Born and Dr. A.E. Hak, for the contribution to patient inclusions, and all the patients that participated in this study.

Disclosure of Interest: None declared

DOI: 10.1136/annrheumdis-2017-eular.5088

\title{
FRI0304 THE UTILITY OF 18F FDG-PET/CT IN DISTINGUISHING BENIGN FROM MALIGNANT RETROPERITONEAL FIBROSIS
}

Y. Wang ${ }^{1}$, Z. Guan ${ }^{2}$, D. Gao ${ }^{1}$, J. Zhu ${ }^{1}$, J. Zhang ${ }^{1}$, F. Huang ${ }^{1} .{ }^{1}$ Rheumatology; ${ }^{2}$ Nuclear Medicine, Chinese PLA General Hospital, Beijing, China

Background: Retroperitoneal fibrosis (RPF) could be idiopathic or secondary to various factors including malignancy. Pathology remains the most reliable diagnostic approach. However, the pathological features have yet been well defined and the feasibility of biopsy procedure depends on several factors such as the proximity of the lesions to abdominal aorta. ${ }^{18} \mathrm{~F}$ FDG-PET/CT may help exclude malignancy by assessing FDG-uptake and mapping the whole body, especially when biopsy procedure is inaccessible.

Objectives: To evaluate the utility of PET/CT in distinguishing benign from malignant RPF.

Methods: Patients with benign or malignant RPF diagnosed between September 2011 and June 2016 were included. Morphologic features, FDG-uptake of retroperitoneal lesions and lymph nodes (LNs) were compared. FDG-uptake of retroperitoneal lesions was assessed visually with the referrence of liver and assessed semiquantitatively with SUVmax. LNs were regarded as specific LNs if frequencies of LNs with high FDG-uptake were observed differently between two forms of RPF. ROC analyses were performed to evaluate the diagnostic accuracy. Results: Seventy-one cases with benign RPF and 21 cases with malignant RPF secondary to lymphoma (15) or metastatic carcinoma (6) with primary sites of pancreas (2), colon (2), stomach (1) and kidney (1) were included. The craniocaudal length, axial width, and distances between anterior or posterior

Table 1. Morphologic features, FDG-uptake of retroperitoneal lesions and lymph nodes (LNs)

\begin{tabular}{lccc}
\hline Parameters & $\begin{array}{c}\text { Benign RPF } \\
(71)\end{array}$ & $\begin{array}{c}\text { Malignant RPF } \\
(21)\end{array}$ & $p$ value \\
\hline
\end{tabular}

Morphologic features

Craniocaudal length, median (IQR), mm

Axial width, median (IQR), mm

Aorta lumen to anterior limit, median (IQR), mm

Aorta lumen to posterior limit, median (IQR), mm

FDG-uptake of retroperitoneal lesions

High FDG-uptake, n (\%)

SUV max, mean (S.D.)

LNs with high FDG-uptake, $\mathrm{n}(\%)$

Hilar/Mediastinal

Cervical

Axillary

Retroperitoneal

Supraclavicular

Inguinal

Peritoneal

Number of specific LNs, $n$ (\%)
$107.0(80.5,136.4) 164.9(104.7,229.2) 0.00$ $41.0(32.3,52.0) \quad 85.0(62.3,98.5) \quad<0.001$ $8.9(6.0,12.0) \quad 20.7(13.9,45.3) \quad<0.001$ $2.8(2.2,4.4)$ $55(77.5) \quad 21(100.0) \quad 0.017$ $\begin{array}{lll}4.8(1.7) & 12.2(7.1) \quad<0.001\end{array}$ $27(38.0) \quad 13(61.9) \quad 0.054$ $\begin{array}{lll}6(8.5) & 5(23.8) & 0.058\end{array}$ $\begin{array}{lll}6(8.5) & 5(23.8) & 0.058 \\ 4(5.6) & 6(28.6) & 0.003\end{array}$ $3(4.2) \quad 16(76.2) \quad<0.001$ $2(2.8) \quad 8(38.1) \quad<0.001$ $1(1.4) \quad 5(23.8) \quad<0.001$ $10(47.6)<0.001$ $\begin{array}{lcc}0(0.0) & 10(47.6) & <0.001 \\ 0(0,0) & 2(1,3) & <0.001\end{array}$

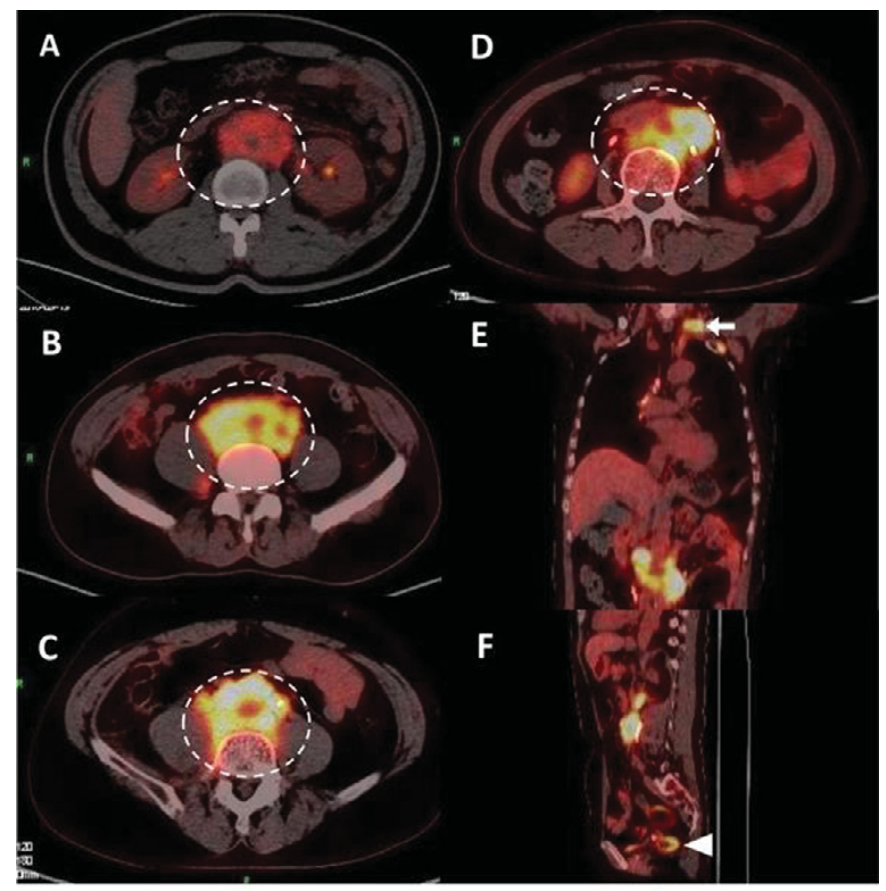

Figure 1. Representative ${ }^{18} \mathrm{~F}$ fluorodeoxyglucose positron emission tomograph images for benign retroperitoneal fibrosis (A) and malignant retroperitoneal fibrosis secondary to lymphoma (B, C) and metastatic carcinoma (D). The retroperitoneal process are encircled by circles in the same size with different level of FDG-uptake. D-F are images from one patient with histological diagnosis of colon cancer. FDG-uptake is accumulated in left supraclavicular lymph node pointed by the arrow (E) and in sigmoid colon pointed by the arrow head $(F)$. 